\title{
A Capacitated Location-Allocation Model for Flood Disaster Service Operations with Border Crossing Passages and Probabilistic Demand Locations
}

\author{
Seyed Ali Mirzapour, ${ }^{1}$ Kuan Yew Wong, ${ }^{1}$ and Kannan Govindan ${ }^{2}$ \\ ${ }^{1}$ Department of Manufacturing and Industrial Engineering, Faculty of Mechanical Engineering, \\ Universiti Teknologi Malaysia, 81310 UTM Skudai, Malaysia \\ ${ }^{2}$ Department of Business and Economics, University of Southern Denmark, 5230 Odense, Denmark
}

Correspondence should be addressed to Kuan Yew Wong; wongky@fkm.utm.my

Received 16 August 2013; Revised 14 October 2013; Accepted 14 October 2013

Academic Editor: Tsan-Ming Choi

Copyright ( 2013 Seyed Ali Mirzapour et al. This is an open access article distributed under the Creative Commons Attribution License, which permits unrestricted use, distribution, and reproduction in any medium, provided the original work is properly cited.

\begin{abstract}
Potential consequences of flood disasters, including severe loss of life and property, induce emergency managers to find the appropriate locations of relief rooms to evacuate people from the origin points to a safe place in order to lessen the possible impact of flood disasters. In this research, a p-center location problem is considered in order to determine the locations of some relief rooms in a city and their corresponding allocation clusters. This study presents a mixed integer nonlinear programming model of a capacitated facility location-allocation problem which simultaneously considers the probabilistic distribution of demand locations and a fixed line barrier in a region. The proposed model aims at minimizing the maximum expected weighted distance from the relief rooms to all the demand regions in order to decrease the evacuation time of people from the affected areas before flood occurrence. A real-world case study has been carried out to examine the effectiveness and applicability of the proposed model.
\end{abstract}

\section{Introduction}

Due to various occurrences of disasters such as floods, hurricanes, and earthquakes which lead to enormous property damages and human injuries, disaster management has recently become a crucial issue. Within disaster management tasks, finding the appropriate locations of relief rooms can help emergency managers to ensure public safety and wellbeing in tragic situations. Thus, transportation specialists play a prominent role in the emergency management process which focuses on people evacuation from disaster events in order to mitigate injury and loss of life. In this regard, there has been an increasing interest among practitioners and scholars in the field of emergency facility location problems. Compared to all disasters, flood is more possible to be predicted and prevented. Therefore, it is vital for flood disaster managers to find the appropriate locations of relief rooms or emergency medical services and allocate them to populations in order to provide efficient services.
A restricted region is a limitation in an area in which the geographic features obstruct the construction of relief rooms. Generally, a restricted planar area is divided into three categories: forbidden regions, congested regions, and regions where neither placement nor travelling is permitted through them. Lakes, mountains, highways, and military zones are some practical examples of these restricted regions. Hamacher and Nickel [1] have performed an extensive review of facility location problems with restrictions. These limitations were incorporated by Klamroth [2] into a model in which a fixed line barrier in a region divides it into two halfplanes and two passages located along the line barrier provide communication between these subregions. The model proposed by Klamroth [2] is a specific formulation, and it may not be appropriate for cases that have regional probability distributions of customers or different objective functions. In the real world, when we are dealing with establishing permanent emergency service facilities for future disasters over a long term planning horizon, it is essential to consider 
the regional probability distribution of customers due to the uncertainties of events and partial information and data. Therefore, a more applicable model which provides better solutions would be beneficial for overcoming this weakness.

To the best of our knowledge, few research activities which consider barriers in emergency facility location problems have been accomplished, due to the computational complexities associated with these problems. Moreover, far too little attention has been paid to developing a practical mathematical model of emergency facility location that considers probabilistic customer regions and a line barrier with border crossings simultaneously. In this research, in order to address the abovementioned problems, we develop a mixed integer nonlinear programming (MINLP) model of a capacitated facility location-allocation problem (CFLAP) in an area in which a fixed line barrier and probabilistic customer regions are taken into account.

The rest of this paper is organized as follows: Section 2 provides a comprehensive literature review on related topics. Section 3 gives an explanation of the problem, and Section 4 presents the proposed mathematical model. In order to show the applicability of the proposed model, a suitable case study is illustrated in Section 5, and the model is validated in Section 6. Finally, Section 7 presents some concluding remarks and outlines a number of future research directions.

\section{Literature Review}

2.1. Emergency Facility Location Problems. The emergency facility location models are categorized into three different types: P-median, P-center, and maximal covering location problems. Since the objective of this research is to design an effective response strategy for disaster management organizations in order to reduce casualties, a planar center location problem (PCLP) would best suit our purpose. Actually, whenever the average cost of servicing the customers is less important than ensuring that no customer receives poor quality of services, the minimax location problem is typically proposed [3]. The procedure of the PCLP model, which is in fact a minimax problem, looks for the center in order to minimize the maximum service time, cost, or loss. Research in this area started with Sylvester [4] which was the first paper that considered the Weber problem with the PCLP model. Elzinga and Hearn [5] then introduced the Euclidean center problem with equal weights and offered an efficient solution technique for their proposed model. Francis et al. [6] made it possible to stress the importance of different customers by assigning unequal weights to them. Mehrez et al. [7] applied a single objective optimization to determine the location of a new hospital. The solutions of this problem were generated by a qualitative approach based on the evaluation of experts' judgments. After that, Daskin et al. [8] proposed an errorbound driven demand point aggregation for the minimax problem with rectilinear distance. Hurtado et al. [9] came out with some constraints on the minimax problem and used a time algorithm to solve it.

In the last two decades, the field of disaster management has gained increasing attention. Many authors have conducted several studies on predisaster preparedness and postdisaster responsiveness, including Altay and Green III [10], Simpson and Hancock [11], Ichoua [12], and Shishebori and Jabalameli [13]. Caunhye et al. [14] and Galindo and Batta [15] performed an extensive review of optimization models in emergency logistics for disaster operations management. Sherali et al. [16] proposed a capacitated facility locationallocation model in order to minimize the congested evacuation time by finding the optimal locations of evacuation shelters under hurricane or flood conditions. Chang et al. [17] studied the problem of locating relief rooms and allocating relief resources for flood emergency preparation. They proposed two stochastic models under possible flood scenarios in which the demand locations are uncertain and depend on the level of flood in different scenarios. In addition, Sheu [18] presented an earthquake relief model that forecasts the demand of affected regions and coordinates the provision of relief supplies. Mete and Zabinsky [19] dealt with an optimization approach for locating and distributing medical supplies under demand and transportation uncertainties for disaster situations. Some other recent relevant studies on this issue include Jiang et al. [20], Rawls and Turnquist [21], Yi et al. [22], Halper and Raghavan [23], Wu et al. [24], Xu et al. [25], and Duran et al. [26].

2.2. Facility Location Problems under Uncertainty. When addressing the strategic decision of establishing permanent facilities to prepare for future disasters over a long term planning horizon, information is uncertain due to the time lag; so considering the location of demands as a known point with certainty leads to poor model performance [27]. The first research in the probabilistic Weber problem was done by Cooper [28] who focused on a single facility location problem. After that, Katz and Cooper [29] approximated the expected distance of transportation via Euclidean distance in which the customer locations were assumed to have a normal distribution. They proposed an algorithm to minimize the total expected distance between the new facility and existing facilities. Later on, for the case of rectilinear distance, Wesolowsky [30] considered three different probability distributions of bivariate normal, bivariate symmetric exponential, and bivariate uniform for customer locations in solving the probabilistic Weber problem.

Although some studies have recently been done for the case of multifacility minimax location problems, much less attention has been given to the probabilistic formulation of minimax problems. Stochastic demand locations were first considered by Carbone and Mehrez [31] for the minimax location problem where the locations of demand points were normally distributed with predetermined means and variances. An emergency location problem with a weighted minimax objective function has been presented by Berman et al. [32] in which the weights associated with each demand point were not given and each of them was assumed to have a uniform distribution. They proved that the objective function is convex for parameters of the uniform distribution, and, thus, the model can be solved by using standard optimization techniques. The minimax regret location problem has been 
investigated by Averbakh and Bereg [33] in the case of uncertain weights and coordinates of demand points. They proposed a model with two objective functions of the median and center problems by using rectilinear distance. Foul [34] applied a mathematical model with the minimax objective function to find the unique location where the demand points follow a bivariate uniform distribution. Then, Pelegrín et al. [35] proposed a comprehensive framework for the 1-center problem where the weights of demands have an arbitrary probability distribution. They have studied a variety of problems using a number of objective functions.

After that, Durmaz et al. [36] presented a model on the probabilistic capacitated multifacility Weber problem in a two-dimensional region by assuming that the locations of customers have some multivariate probability distributions. Later on, Canbolat and Von Massow [37] shared their work on locating emergency facilities with random demands. They proposed an approach that explicitly addresses the uncertainty with respect to where an emergency will occur by minimizing the maximum risk through the minimization of the maximum expected distance. Then, Hosseinijou and Bashiri [38] formulated an appropriate model to represent the minimax single facility location problem in which demand areas are weighted and their coordinates have a bivariate uniform distribution. They proposed a model which finds the optimal location of the transfer point such that the maximum expected weighted distance from the facility point to all demand points through the transfer point is minimized.

2.3. Facility Location Problems with Barriers. In many cases of facility location problems, difficulties in solution methodologies occur when barriers limit the establishment of new facilities in a specified region. The Weber problem with a circular barrier was first presented by Katz and Cooper [39]. Since then, researchers have studied a variety of location problems in the presence of barriers using different types of objective function. They showed that the objective functions of these problems are nonconvex and offered some heuristic methods for solving them. Klamroth and Wiecek [40] focused on solving a multiobjective median problem (MOMP) with the minisum objective function by assuming a line barrier in an area and showed that their proposed model is nonconvex. Many researchers have applied different heuristic algorithms for the Weber problem that considers the convex or nonconvex polyhedral barriers in a region (e.g., [41-43]). The location problem has also been extended by Klamroth [44] who suggested that the single circular barrier can be subdivided into some feasible convex regions. By using this method, the nonconvex objective function of the problem has been transformed into some convex objective functions in each region. When the number of subdivided convex regions increases, this method is not suitable, since dividing the convex regions becomes awkward. To overcome this complexity, a genetic algorithm has been proposed by Bischoff and Klamroth [45] based on the Weiszfeld technique to solve the model with a large number of demand points. Bischoff et al. [46] presented two alternative locationallocation heuristics for the multifacility Weber problem with barriers.

The locations of optimum points for a given number of passages in two cases of capacitated and incapacitated facility location problems have been discussed by Huang et al. [48]; their model tried to minimize the transportation cost. Increasingly, more authors have addressed the planar center location problem in the presence of barriers in which the objective function is to find a facility that minimizes the maximum distance (e.g., [49-51]). A different perspective of using a wave front approach was proposed by Frieß et al. [52] for the minimax location problem in the presence of barriers. Canbolat and Wesolowsky [53] developed a model by considering a probabilistic line barrier that occurs randomly on the horizontal route. Their objective was to locate a single new facility to minimize the sum of the expected distances from all customer locations. After that, Canbolat and Wesolowsky [54] presented a new experimental approach for the Weber problem by using the Varignon frame. Their proposed method tried to minimize the sum of the weighted distances from the facility to customer points by considering barriers in a region. Then, Canbolat and Wesolowsky [47] presented a planar single facility location problem in the presence of border crossing. They developed the models for minisum and minimax problems in the presence of a fixed line barrier which divides a region into two subregions.

In short, although previous studies have proposed different approaches to address the facility location-allocation problem in the presence of a barrier, they have somehow neglected the probabilistic nature of customer locations. Moreover, very limited research activities have been carried out on multifacility location problems; albeit in many realworld applications, more than a single facility is required to be established. Therefore, an appropriate mathematical model is proposed in this paper in order to address these issues.

\section{Problem Definition and Formulation}

3.1. Facility Location Problem in the Presence of a Line Barrier. It is important to introduce some preliminary definitions before formally defining the problem. Based on Klamroth [2], the facility location problem in the presence of a line barrier is described as follows.

Let $L$ be a line and $\left\{P_{k}=\left(x^{k}, y^{k}\right) \epsilon L \mid k \in K:=\{1, \ldots, K\}\right\}$ be a set of points on $L$. Then, $B_{L}:=L \backslash\left\{P_{1}, \ldots, P_{k}\right\}$ is called a fixed line barrier with passages. The feasible region $\theta$ is defined as the union of two closed half-planes $\theta_{1}$ and $\theta_{2}$ on both sides of $B_{L} \cdot P_{k}=\left(x^{k}, y^{k}\right)$ is the coordinate of a border crossing passage. Since the connections are located on a fixed horizontal line barrier, we set $y^{k}=\varphi, k=1, \ldots, K$. A set of existing demand regions with locations of $X_{j}, j=1, \ldots, J$ and positive weights of $w_{j}$ is given in $\theta$. Then, $d_{p}^{B}\left(X_{i}, X_{j}\right)$ is the p-norm barrier distance between $X_{i}$ and $X_{j}$ in the presence of a fixed line barrier where communication between two 
subplanes is allowed only through the $K$ passages. The basic minimax model with barriers can be stated as follows:

$$
\begin{array}{r}
\operatorname{Min} F\left(X_{i}\right)=\operatorname{Max}\left\{w_{j} \cdot d_{p}^{B}\left(X_{i}, X_{j}\right)\right\}, \\
i=1, \ldots, I, \quad j=1, \ldots, J
\end{array}
$$

Any demand region that is in the same half-plane will not be affected by the presence of the barrier. The other demand regions that are not in the same half-plane with the new facility will connect to the new facility through just one of the passages along the line barrier. For this problem, the rectilinear distance, $p=1$, is considered to compute the distance between the new emergency facilities and all demand regions.

\subsection{Stochastic Weighted Emergency Facility Location Problem} in the Presence of a Line Barrier. Assume that each demand region $j$ has a coordinate of $X_{j}=\left(U_{j}, V_{j}\right)$ such that $U_{j}, V_{j}$ are independent random variables and each of them has a bivariate uniform distribution. This assumption is realistic in real-world cases wherein the geographical distribution of population in different regions is uniform in square or rectangular areas [38]. Furthermore, a uniform distribution provides a "building block" to deal with a situation which is not precisely approximated [34].

The problem is to find the optimum location of the new facility $\left(x_{i}{ }^{*}, y_{i}{ }^{*}\right)$ such that the maximum expected weighted distance from the facility to all demand regions, $E F\left(X_{i}{ }^{*}\right)$, is minimized:

$$
\begin{array}{r}
\operatorname{Min} E F\left(X_{i}^{*}\right)=\operatorname{Max}\left\{E\left[w_{j} \cdot d_{1}^{B}\left(\left(x_{i}{ }^{*}, y_{i}{ }^{*}\right),\left(U_{j}, V_{j}\right)\right)\right]\right\} \\
=\operatorname{Max}\left\{w_{j} \cdot E\left[d_{1}^{B}\left(\left(x_{i}{ }^{*}, y_{i}{ }^{*}\right),\left(U_{j}, V_{j}\right)\right)\right]\right\} \\
i=1, \ldots, I, j=1, \ldots, J,
\end{array}
$$

where $E[\cdot]$ represents the expected value. The above mentioned problem is illustrated in Figure 1. The weights of emergency needs in each demand region are defined as a function of two factors. The first factor is related to the qualitative criterion of demand region $j$ which can be the quality of road construction from a demand region to the new facility point whereas the next one is concerned with the quantitative criterion of demand region $j$ which can be a function of population, potential of emergency situation occurrence, and other issues. The weight $Q_{j}$ of the qualitative factor has an inverse relation with $w_{j}$ which means that $w_{j}$ will reduce whenever the quality of road construction increases and this causes the new facility to be located farther from demand region $j$. The weight $P_{j}$ of the quantitative factor has a direct relation with $w_{j}$; for instance, whenever the population of demand region $j$ increases, $w_{j}$ will increase too and the new emergency facility will tend to be located nearer to this customer area. In order to use both quantitative and qualitative factors in the total weight of demand region $j$, they must be defined in the same range to be comparable. One method of doing so is normalizing both types of factors to be in the domain of zero to one. Based on the experience

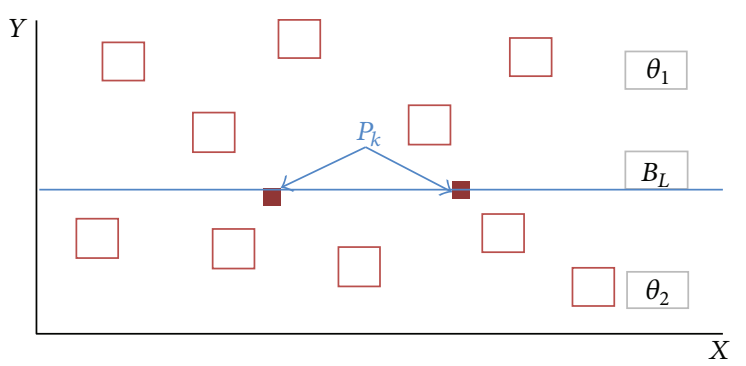

FIgURE 1: Demand locations with a fixed line barrier and two passages on the plane.

of emergency managers, both quantitative and qualitative factors have the same significance.

In this paper, the case of uniformly distributed demand regions in several rectangles is considered. It uses the rectilinear distance concept for measuring distances. Imagine a number of demand regions $j$ in which $U_{j}, V_{j}$ are independent random variables in $\left[a_{j}, b_{j}\right]$ and $\left[c_{j}, d_{j}\right]$ with probability density functions $f_{u}\left(U_{j}\right)=1 /\left(b_{j}-a_{j}\right)$ and $f_{v}\left(V_{j}\right)=1 /\left(d_{j}-\right.$ $c_{j}$ ), respectively. Each area is a demand region, and it is desirable to find the optimum locations of emergency services among all these regions. The objective is to find the optimal locations of emergency facilities to provide efficient services for people. The mathematical expression for the expected distance between new facility $i$ and demand region $j$ is presented as

$$
\begin{aligned}
E\left[d\left(X_{i}, X_{j}\right)\right] & =E\left[\left|U_{j}-x_{i}\right|+\left|V_{j}-y_{i}\right|\right] \\
& =E\left[\left|U_{j}-x_{i}\right|\right]+E\left[\left|V_{j}-y_{i}\right|\right] .
\end{aligned}
$$

Let $E\left[\left|U_{j}-x_{i}\right|\right]$ and $E\left[\left|V_{j}-y_{i}\right|\right]$ be denoted by $H_{i}(x)$ and $G_{i}(y)$, respectively. Then, we have

$$
\begin{aligned}
H_{i}(x) & =E\left[\left|U_{j}-x_{i}\right|\right]=\int_{-\infty}^{\infty}\left|U_{j}-x_{i}\right| \cdot f_{u}\left(U_{j}\right) d_{u} \\
& =\frac{1}{b_{j}-a_{j}} \int_{a_{j}}^{b_{j}}\left|U_{j}-x_{i}\right| d_{u}, \\
G_{i}(y) & =E\left[\left|V_{j}-y_{i}\right|\right]=\int_{-\infty}^{\infty}\left|V_{j}-y_{i}\right| \cdot f_{v}\left(V_{j}\right) d_{v} \\
& =\frac{1}{d_{j}-c_{j}} \int_{c_{j}}^{d_{j}}\left|V_{j}-y_{i}\right| d_{v} .
\end{aligned}
$$

The closed-form expression for the expected distance $E\left[d\left(X_{i}, X_{j}\right)\right]$ was developed by Foul [34], which can be written as

$$
H_{i}(x)= \begin{cases}\frac{-x_{i}+\frac{a_{j}+b_{j}}{2}}{\left(x_{i}-a_{j}\right)^{2}+\left(x_{i}-b_{j}\right)^{2}} & \text { if } x_{i} \leq a_{j} \\ 2 \cdot\left(b_{j}-a_{j}\right) & \text { if } a_{j} \leq x_{i} \leq b_{j} \\ x_{i}-\frac{a_{j}+b_{j}}{2} & \text { if } x_{i} \geq b_{j} .\end{cases}
$$


$G_{i}(y)$ can be defined like $H_{i}(x)$ by substituting $x_{i}, a_{j}, b_{j}$ with $y_{i}, c_{j}, d_{j}$, respectively. With this expression, the problem is formulated as follows:

$$
\begin{array}{r}
\operatorname{Min} E F\left(X_{i}\right)=\operatorname{Max}\left\{w_{j} \cdot\left[H_{i}(x)+G_{i}(y)\right]\right\}, \\
i=1, \ldots, I, j=1, \ldots, J .
\end{array}
$$

Equation (7) can be transformed into an equivalent constrained nonlinear program which is presented here:

$$
\begin{array}{ll}
\text { Min } & Z \\
\text { Subject to } & Z \geq w_{j} \cdot\left[H_{i}(x)+G_{i}(y)\right], \\
& i=1, \ldots, I, j=1, \ldots, J .
\end{array}
$$

\section{Proposed Mathematical Model}

In this section, a MINLP model is proposed for CFLAP. The proposed MINLP model aims at finding the suitable locations of some emergency facilities in the presence of a fixed line barrier such that the expected weighted rectilinear distance between the emergency facilities and the farthest demand regions is minimized. The following assumptions and notations are used in formulating the MINLP model for CFLAP.

Assumptions. (i) The demand regions' coordinates have a bivariate uniform distribution because customer populations (residential areas) are normally dispersed or structured in square or rectangular areas.

(ii) There is a line barrier in the area which is fixed.

(iii) Two emergency service facilities are required to be established. This will ensure a more balanced allocation of the demand regions to the emergency service facilities since the entire region is divided into two half-planes by a line barrier.

\section{Notations}

\section{I: Number of new facilities}

$J$ : Number of demand regions or customer regions

$K$ : Number of passages

$w_{j}$ : Positive weight of each existing demand region $j$

$t_{i j}$ : Binary variable: 1 , if demand region $j$ is assigned to new facility $i, 0$, otherwise

$h_{i j k}$ : Binary variable: 1 , if demand region $j$ is served by new facility $i$ through passage $k, 0$, otherwise

$l_{i j}$ : Binary variable: 1 , if demand region $j$ and new facility $i$ are located in different half-planes, 0 , otherwise

$g_{i}$ : Binary variable: 1 , if new facility $i$ is located in the upper half-plane, 0 , otherwise

$q_{j}$ : Binary parameter: 1 , if demand region $j$ is located in the upper half-plane, 0 , otherwise

$C a_{i}$ : Maximum capacity of new facility $i$

$S 1_{i j}$ : Binary variable: 1 , if $x_{i} \leq a_{j}, 0$, otherwise

$S 2_{i j}$ : Binary variable: 1 , if $a_{j}<x_{i}<b_{j}, 0$, otherwise

$S 3_{i j}$ : Binary variable: 1 , if $x_{i} \geq b_{j}$, 0 , otherwise
$F 1_{i j}$ : Binary variable: 1 , if $y_{i} \leq c_{j}, 0$, otherwise

$F 2_{i j}$ : Binary variable: 1 , if $c_{j}<y_{i}<d_{j}$, 0 , otherwise

$F 3_{i j}$ : Binary variable: 1 , if $y_{i} \geq d_{j}, 0$, otherwise

$S 1_{j k}$ : Binary variable: 1 , if $x^{k} \leq a_{j}, 0$, otherwise

$S 2_{j k}$ : Binary variable: 1 , if $a_{j}<x^{k}<b_{j}, 0$, otherwise

$S 3_{j k}$ : Binary variable: 1 , if $x^{k} \geq b_{j}, 0$, otherwise

$F 1_{j}$ : Binary variable: 1 , if $\varphi \leq c_{j}, 0$, otherwise

$F 2_{j}$ : Binary variable: 1 , if $c_{j}<\varphi<d_{j}, 0$, otherwise

$F 3_{j}$ : Binary variable: 1 , if $\varphi \geq d_{j}$, 0 , otherwise.

Based on the above assumptions and notations, the objective function and constraints will be described.

4.1. Objective Function. The objective function tries to minimize $Z$ while satisfying constraint (9). Since $Z$ needs to be minimized, the shortest possible path through one of the border crossings along the barrier will be selected. The objective function can be formed as follows:

$$
\begin{aligned}
\text { Min } Z & \\
\text { where } Z \geq w_{j} \cdot t_{i j} \cdot[ & {\left[\left(\sum _ { k = 1 } ^ { K } \left[T_{k}\left(x^{k}\right)+R_{k}\left(y^{k}\right)+\left|x^{k}-x_{i}\right|\right.\right.\right.} \\
& \left.\left.\left.+\left|y^{k}-y_{i}\right|\right] \cdot h_{i j k}\right) \cdot l_{i j}\right] \\
& \left.+\left[\left[H_{i}(x)+G_{i}(y)\right] \cdot\left(1-l_{i j}\right)\right]\right] .
\end{aligned}
$$

Note that the expected distance from the passage coordinates along the line barrier to the demand regions is denoted by $T_{k}\left(x^{k}\right)$ and $R_{k}\left(y^{k}\right)$ which are equal to $E\left[\left|U_{j}-x^{k}\right|\right]$ and $E\left[\left|V_{j}-y^{k}\right|\right]$ and they can be defined like $H_{i}(x)$ and $G_{i}(y)$ by substituting $x_{i}$ with $x^{k}$ and $y_{i}$ with $y^{k}$, respectively.

4.2. Constraints. The constraints of this problem are formulated as follows.

4.2.1. Expected Distance. Based on the obtained closed-form expression in (6), the expected distance is computed in different ways depending on the location of the new facility and the interval of demand regions. Since this model will be solved by the GAMS software (full academic version 22.1), it is required to convert the formulas of $H_{i}(x)$ into a few constraints by introducing new binary variables of $S 1_{i j}, S 2_{i j}$, and $S 3_{i j}$. This makes it easier for the software to perform the computation process. Likewise, new binary variables of $F 1_{i j}$, $F 2_{i j}$, and $F 3_{i j}$ for $G_{i}(y)$ are introduced. $T_{k}\left(x^{k}\right)$ and $R_{k}\left(y^{k}\right)$ can be manipulated like $H_{i}(x)$ and $G_{i}(y)$ by replacing $S 1_{i j}, S 2_{i j}$, 
$S 3_{i j}$ with $S 1_{j k}, S \dot{2}_{j k}, S \hat{3}_{j k}$ and $F 1_{i j}, F 2_{i j}, F 3_{i j}$ with $F 1_{j}, F \dot{2}_{j}$, $F 3_{j}$, respectively. These constraints can be written as follows:

$$
\begin{gathered}
2 x_{i} \cdot S 1_{i j}-2 a_{j} \cdot S 1_{i j} \leq x_{i}-a_{j} \quad \forall i, j, \\
2 x_{i} \cdot S 3_{i j}-2 b_{j} \cdot S 3_{i j} \geq x_{i}-b_{j} \quad \forall i, j, \\
S 1_{i j}+S 2_{i j}+S 3_{i j}=1 \quad \forall i, j, \\
2 y_{i} \cdot F 1_{i j}-2 c_{j} \cdot F 1_{i j} \leq y_{i}-c_{j} \quad \forall i, j, \\
2 y_{i} \cdot F 3_{i j}-2 d_{j} \cdot F 3_{i j} \geq y_{i}-d_{j} \quad \forall i, j, \\
F 1_{i j}+F 2_{i j}+F 3_{i j}=1 \quad \forall i, j, \\
2 x^{k} \cdot S 1_{j k}-2 a_{j} \cdot S 1_{j k} \leq x^{k}-a_{j} \quad \forall j, k, \\
2 x^{k} \cdot S 3_{j k}-2 b_{j} \cdot S 3_{j k} \geq x^{k}-b_{j} \quad \forall j, k, \\
S 1_{j k}+S 2_{j k}+S 3_{j k}=1 \quad \forall j, k, \\
2 \varphi \cdot F 1_{j}-2 c_{j} \cdot F 1_{j} \leq \varphi-c_{j} \quad \forall j, \\
2 \varphi \cdot F 3_{j}-2 d_{j} \cdot F 3_{j} \geq \varphi-d \quad \forall j, \\
F 1_{j}+F 2_{j}+F 3_{j}=1 \quad \forall j .
\end{gathered}
$$

The first three constraints (constraints (10), (11), and (12)) are related to $H_{i}(x)$. Whenever the variable $x_{i}$ is less than $a_{j}\left(x_{i} \leq a_{j}\right)$, the binary variable $S 1_{i j}$ in constraint (10) will be equal to one; otherwise, it makes the binary variable to be equal to zero. Constraint (11) ensures that the expected distances between facilities and demand regions are calculated by the third formula of (6) whenever the variable $x_{i}$ is more than $b_{j}\left(x_{i} \geq b_{j}\right)$; otherwise $S 3_{i j}$ will be equal to zero. If neither $S 1_{i j}$ nor $S 3_{i j}$ is equal to one, then the binary variable $S 2_{i j}$ must be equal to one due to constraint (12). Constraint (12) also guarantees that just one of these cases can occur for the same new facility and customer region; assignment of two or three binary variables to one will lead to infeasibility and is not allowed. The rest of the constraints (constraints (13) to (21)) follow the same interpretation for $G_{i}(y), T_{k}\left(x^{k}\right)$, and $R_{k}\left(y^{k}\right)$, respectively.

4.2.2. The Position of New Facilities and Customer Regions. Constraint (22) indicates whether the new facility is located in the upper half-plane or not:

$$
2 g_{i} \cdot y_{i}-y_{i} \geq 2 g_{i} \cdot \varphi-\varphi, \quad \forall i
$$

Constraint (23) shows whether the customer region and the new facility are both located in the same half-plane or not:

$$
l_{i j}=\left|q_{j}-g_{i}\right|, \quad \forall i, j .
$$

Constraints (22) and (23) help the objective function to compute the expected distance between the new facilities and the customer regions in different cases.
4.2.3. Demand Allocation. According to constraint (24), each demand region must be served just by one of the new facilities:

$$
\sum_{i=1}^{I} t_{i j}=1, \quad \forall j .
$$

The next constraint assures that the shortest path through just one of the passages will be selected to serve the allocated customer region in a different half-plane:

$$
\sum_{k=1}^{K} h_{i j k}=l_{i j}, \quad \forall i, j .
$$

4.2.4. Capacity. Constraint (26) guarantees that each new facility cannot serve more than its corresponding capacity:

$$
\sum_{j=1}^{J} w_{j} \cdot t_{i j} \leq C a_{i}, \quad \forall i .
$$

The modified objective function with respect to constraints (10) to (21) looks as follows:

Min $Z$

where $Z \geq w_{j} \cdot t_{i j}$

$$
\begin{aligned}
& {\left[\left(\left[\sum _ { k = 1 } ^ { K } \left[\left(-x^{k}+\frac{a_{j}+b_{j}}{2}\right) \cdot S i_{j k}\right.\right.\right.\right.} \\
& +\left(\frac{\left(x^{k}-a_{j}\right)^{2}+\left(x^{k}-b_{j}\right)^{2}}{2 \cdot\left(b_{j}-a_{j}\right)}\right) \cdot S 2_{j k} \\
& +\left(x^{k}-\frac{a_{j}+b_{j}}{2}\right) \cdot S \dot{3}_{j k} \\
& +\left(-y^{k}+\frac{c_{j}+d_{j}}{2}\right) \cdot F 1_{j} \\
& +\left(\frac{\left(y^{k}-c_{j}\right)^{2}+\left(y^{k}-d_{j}\right)^{2}}{2 \cdot\left(d_{j}-c_{j}\right)}\right) \cdot F 2_{j} \\
& +\left(y^{k}-\frac{c_{j}+d_{j}}{2}\right) \cdot F \dot{3}_{j} \\
& \left.\left.\left.+\left|x^{k}-x_{i}\right|+\left|y^{k}-y_{i}\right|\right] \cdot h_{i j k}\right] \cdot l_{i j}\right) \\
& +\left(\left[\left(-x_{i}+\frac{a_{j}+b_{j}}{2}\right) \cdot S 1_{i j}\right.\right. \\
& +\left(\frac{\left(x_{i}-a_{j}\right)^{2}+\left(x_{i}-b_{j}\right)^{2}}{2 \cdot\left(b_{j}-a_{j}\right)}\right) \cdot S 2_{i j} \\
& +\left(x_{i}-\frac{a_{j}+b_{j}}{2}\right) \cdot S 3_{i j} \\
& +\left(-y_{i}+\frac{c_{j}+d_{j}}{2}\right) \cdot F 1_{i j} \\
& +\left(\frac{\left(y_{i}-c_{j}\right)^{2}+\left(y_{i}-d_{j}\right)^{2}}{2 \cdot\left(d_{j}-c_{j}\right)}\right) \cdot F 2_{i j} \\
& \left.\left.\left.+\left(y_{i}-\frac{c_{j}+d_{j}}{2}\right) \cdot F 3_{i j}\right] \cdot\left(1-l_{i j}\right)\right)\right] \text {. }
\end{aligned}
$$




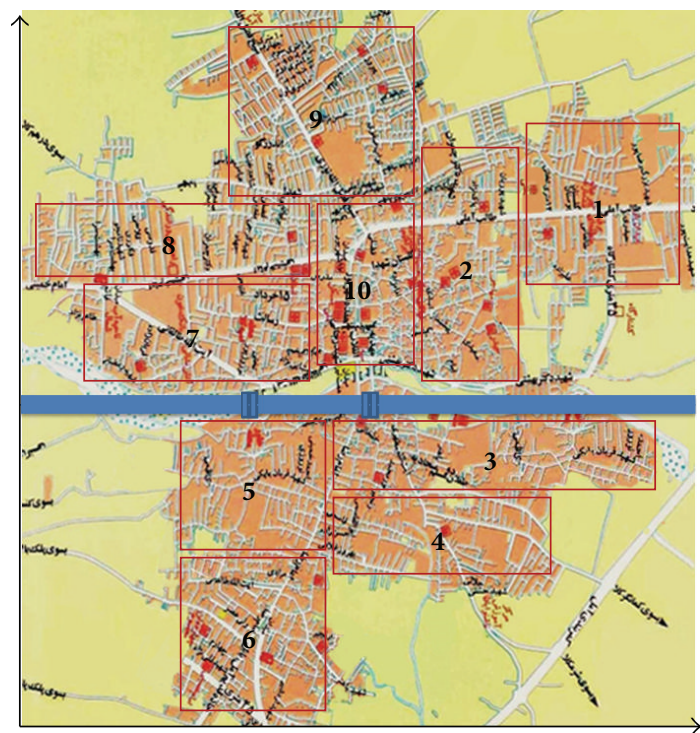

FIgURE 2: The illustration of the demand regions and passages.

\section{A Case Illustration}

To examine the practicality and effectiveness of the proposed model for CFLAP, a case is illustrated for the emergency facility location problem. One of the flood disaster management activities in the Amol city, Mazandaran province, northern part of Iran, was selected as a case study. In recent years, severe floods have occurred in the northern part of Iran. In 1999, floods in this area left 34 dead and 15 missing after more than a week and presumed dead. More than 100 people were injured and various areas were damaged with estimated losses of 15 million dollars [55]. Recently, the frequency of flood occurrence in the Mazandaran province, Iran, has increased and an appropriate model is required to find the suitable locations of relief rooms in order to decrease the evacuation time of people from the affected areas to a safe place before flood occurrence to reduce the possible impact of flood disasters.

The Amol city is located at $36^{\circ} 23^{\prime} \mathrm{N}$ latitude and $52^{\circ} 20^{\prime} \mathrm{E}$ longitude in northern Iran. This city is situated on the Haraz river bank, and it is less than 20 kilometers south of the Caspian sea and 10 kilometers north of the Alborz mountain. Based on the 2010 census, its population was around 224,000 with 61,085 families. As shown in Figure 2, the Haraz river divides the city into two subplanes, and two bridges are located along the river to connect the two subregions with each other. With respect to the geographic information system (GIS) map of the city, it has been divided into ten demand areas. As we mentioned before, the weights of emergency needs in each demand region are a function of both qualitative and quantitative criteria. The population of each area, as a quantitative criterion, was obtained from the statistics center of Iran and is shown in Table 1; the data for quality of road construction were gained from the GIS map of Amol which was prepared by the Department of Housing and Urban Development of Mazandaran Province. Table 2 presents the data for the qualitative factor.
TABLE 1: Population data in different regions.

\begin{tabular}{lcc}
\hline$j$ & Population & $P_{j}$ \\
\hline 1 & 13464 & 0.057132 \\
2 & 26139 & 0.110916 \\
3 & 13984 & 0.059339 \\
4 & 25432 & 0.107916 \\
5 & 23916 & 0.101483 \\
6 & 8888 & 0.037715 \\
7 & 12972 & 0.055044 \\
8 & 35072 & 0.148822 \\
9 & 38392 & 0.162910 \\
10 & 37405 & 0.158722 \\
\hline
\end{tabular}

For the sake of normalizing the quantitative weight $P_{j}$ of each demand region, the population number of each area is divided by the total population. For the qualitative factor, the quality of road construction is categorized into 5 groups which are high quality (HQ), good quality (GQ), acceptable quality (AQ), minor repair required (MNR), and major repair required (MJR). Since this factor is inversely related with $w_{j}$, the best quality construction receives the significance of 1 and the worst quality construction gets the maximum significance score which is equal to 5 . The weight $Q_{j}$ of the qualitative factor is computed similarly as the quantitative criterion. Since the significance of both criteria is the same, the final weight of each area is obtained by adding together half of the weights of the qualitative and quantitative factors.

The coordinates of ten demand regions with their corresponding final weights are tabulated in Table 3, while Table 4 gives the coordinates of the passages. It is noted that the establishment of two relief rooms with a maximum capacity percentage of 0.545 and 0.46 is required to cover all the demand regions. The proposed MINLP model contains 292 integer variables and 4 noninteger variables in terms of its complexity. Hence, it has been implemented in the GAMS 22.1 software using the BARON solver in order to identify the optimal values of $x_{i}^{*}$ and $y_{i}^{*}$. BARON was created at the University of Illinois which implements global optimization algorithms of a branch and bound type and solves nonconvex problems to obtain global solutions [56]. Computations were performed using a system with a Core 2 duo $2.1 \mathrm{GHz} \mathrm{CPU}$ and $2 \mathrm{~GB}$ RAM and the computation time for solving the model was about 11 minutes. The time complexity grows exponentially with the increasing number of passages or new facilities.

The optimal locations of the emergency facilities and the value of the objective function are reported in Table 5. In addition, Table 6 shows the optimal corresponding allocation clusters of the two new emergency facilities. For a better understanding, Figure 3 illustrates the optimum locations of the new facilities and their corresponding allocation. Based on the results, we recommend that demand regions $1,2,8$, 9, and 10 are served by the first new facility and the rest of the demand areas are served by the second new facility. The results will be useful for emergency managers to decrease the evacuation time in affected areas before flood occurrence. 
TABLE 2: Data for quality of road construction.

\begin{tabular}{lcccccrr}
\hline$j$ & HQ (\%) & GQ (\%) & AQ (\%) & MNR (\%) & MJR (\%) & Score (\%) of each area \\
\hline 1 & 0.38 & 40.91 & 37.5 & 20.19 & 0 & 275.46 & 281.62 \\
2 & 1.65 & 39.76 & 35.76 & 11.53 & 9.41 & 382.32 & 0.089512 \\
3 & 0 & 11.76 & 28.43 & 25.49 & 34.31 & 388.61 & 345.89 \\
4 & 1.37 & 6.65 & 23.29 & 39.33 & 29.35 & 357.5 & 0.124236 \\
5 & 2.96 & 20 & 28.89 & 24.44 & 23.7 & 240.09 & 0.126280 \\
6 & 0.72 & 11.15 & 40.66 & 24.75 & 22.7 & 0.112398 \\
7 & 2.78 & 59.45 & 30.87 & 6.45 & 0 & 224.59 & 0.078018 \\
8 & 5.97 & 68.1 & 20.71 & 3.36 & 1.37 & 0.072981 \\
9 & 1.54 & 70.51 & 26.15 & 1.79 & 0 & 35.17 & 0.074145 \\
10 & 15.79 & 14.04 & 14.04 & 10.53 & 45 & 0.114744 \\
\hline
\end{tabular}

TABLE 3: Parameter values for the demand regions.

\begin{tabular}{cccc}
\hline$j$ & {$\left[a_{j}, b_{j}\right]$} & {$\left[c_{j}, d_{j}\right]$} & $w_{j}$ \\
\hline 1 & {$[14.6,19.1]$} & {$[11.4,17.1]$} & 0.073322 \\
2 & {$[11.4,14.5]$} & {$[8.9,15]$} & 0.101215 \\
3 & {$[9.2,18.2]$} & {$[5.9,8]$} & 0.0917875 \\
4 & {$[9.2,15.4]$} & {$[3.8,5.8]$} & 0.117098 \\
5 & {$[4.7,9.1]$} & {$[4.7,8]$} & 0.1069405 \\
6 & {$[4.7,9.1]$} & {$[0.3,4.6]$} & 0.076943 \\
7 & {$[1.9,8.4]$} & {$[8.9,11.4]$} & 0.066531 \\
8 & {$[0.5,8.4]$} & {$[11.5,13.6]$} & 0.1109015 \\
9 & {$[6.2,11.3]$} & {$[13.7,18.2]$} & 0.1185275 \\
10 & {$[8.6,11.3]$} & {$[9.4,13.6]$} & 0.136733 \\
\hline
\end{tabular}

TABLE 4: The coordinates of passages.

\begin{tabular}{lcc}
\hline$k$ & $x^{k}$ & $y^{k}$ \\
\hline 1 & 6.9 & 8.5 \\
2 & 10.1 & 8.5 \\
\hline
\end{tabular}

TABLE 5: Optimal solutions for the locations of relief rooms.

\begin{tabular}{ccccc}
\hline$x_{1}{ }^{*}$ & $y_{1}{ }^{*}$ & $x_{2}{ }^{*}$ & $y_{2}{ }^{*}$ & $F\left(X_{i}{ }^{*}\right)$ \\
\hline 9.8 & 13.312 & 8.6 & 6.139 & 0.682 \\
\hline
\end{tabular}

TABLE 6: Optimal allocation of demand areas to the two new facilities.

\begin{tabular}{lccc}
\hline$(i, j)$ & $t_{i j}$ & $(i, j)$ & $t_{i j}$ \\
\hline 1,1 & 1 & 2,1 & 0 \\
1,2 & 1 & 2,2 & 0 \\
1,3 & 0 & 2,3 & 1 \\
1,4 & 0 & 2,4 & 1 \\
1,5 & 0 & 2,5 & 1 \\
1,6 & 0 & 2,6 & 1 \\
1,7 & 0 & 2,7 & 1 \\
1,8 & 1 & 2,8 & 0 \\
1,9 & 1 & 2,9 & 0 \\
1,10 & 1 & 2,10 & 0 \\
\hline
\end{tabular}

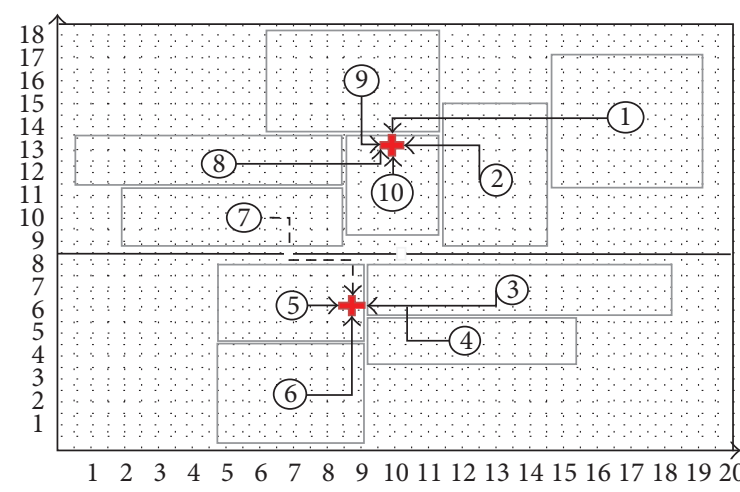

FIGURE 3: Illustration of the computation results.

TABLE 7: Coordinates of randomly selected areas.

\begin{tabular}{cccc}
\hline$j$ & {$\left[a_{j}, b_{j}\right]$} & {$\left[c_{j}, d_{j}\right]$} & $w_{j}$ \\
\hline 1 & {$[14.6,19.1]$} & {$[11.4,17.1]$} & 0.159 \\
3 & {$[9.2,18.2]$} & {$[5.9,8]$} & 0.198 \\
5 & {$[4.7,9.1]$} & {$[4.7,8]$} & 0.234 \\
7 & {$[1.9,8.4]$} & {$[8.9,11.4]$} & 0.145 \\
9 & {$[6.2,11.3]$} & {$[13.7,18.2]$} & 0.264 \\
\hline
\end{tabular}

\section{Validation of the Model}

Model validation is essential for evaluating the proposed model to make sure that the chosen new facility locations truly minimize the maximum value of distance from the stochastic demand regions to the chosen new facility points within the defined area. Based on the validation procedure of Hosseinijou and Bashiri [38], ten demand points were randomly generated within each demand region. Then, the objective functions of the recommended emergency facility locations were compared with the objective function of the proposed model.

Some efforts of validating the model are presented here. Ten scenarios were created from five randomly selected areas in Table 3. A random data toolbox in the Minitab software was used for generating random point data in each area. The weight of each point in the scenarios is equal to the weight of 
TABLE 8: Validation results of the proposed model.

\begin{tabular}{|c|c|c|c|c|c|c|c|}
\hline Scenario & $\begin{array}{c}X_{1}=\left(U_{1}, V_{1}\right) \\
{[14.6,19.1]} \\
\times[11.4,17.1]\end{array}$ & $\begin{array}{c}X_{3}=\left(U_{3}, V_{3}\right) \\
{[9.2,18.2]} \\
\quad \times[5.9,8]\end{array}$ & $\begin{array}{c}X_{5}=\left(U_{5}, V_{5}\right) \\
\quad[4.7,9.1] \\
\quad \times[4.7,8]\end{array}$ & $\begin{array}{c}X_{7}=\left(U_{7}, V_{7}\right) \\
{[1.9,8.4]} \\
\times[8.9,11.4]\end{array}$ & $\begin{array}{c}X_{9}=\left(U_{9}, V_{9}\right) \\
{[6.2,11.3]} \\
\times[13.7,18.2]\end{array}$ & $F\left(X_{i}^{*}\right)$ & |Error| \\
\hline 1 & $(18.43,16.78)$ & $(12.05,7.74)$ & $(7.96,7.07)$ & $(5.26,11.26)$ & $(7.95,15.57)$ & 1.160 & 0.05 \\
\hline 2 & $(19.10,13.44)$ & $(13.65,6.13)$ & $(8.78,7.62)$ & $(3.75,9.69)$ & $(10.96,16.86)$ & 1.147 & 0.037 \\
\hline 3 & $(16.57,16.69)$ & $(10.67,7.55)$ & $(4.86,5.87)$ & $(7.05,9.27)$ & $(7.69,14.57)$ & 1.092 & 0.018 \\
\hline 4 & $(17.42,12.74)$ & $(13.48,7.8)$ & $(5.46,6.5)$ & $(3.45,10.45)$ & $(9.93,16.23)$ & 1.189 & 0.079 \\
\hline 5 & $(16.67,15.29)$ & $(10.71,7.57)$ & $(5.03,7.44)$ & $(2.35,9.89)$ & $(7.46,13.93)$ & 1.049 & 0.061 \\
\hline 6 & $(14.97,12.32)$ & $(15.95,6.95)$ & $(4.97,4.84)$ & $(2.66,10.68)$ & $(10.47,17.96)$ & 1.425 & 0.315 \\
\hline 7 & $(15.66,14.60)$ & $(12.09,7)$ & $(5.16,7)$ & $(6.85,10.15)$ & $(7.5,17.14)$ & 1.062 & 0.048 \\
\hline 8 & $(16.55,13.11)$ & $(13.51,7.06)$ & $(8.99,7)$ & $(2.17,9.81)$ & $(10.69,16.83)$ & 1.179 & 0.069 \\
\hline 9 & $(16.30,14.64)$ & $(16.65,6.87)$ & $(6.55,7.44)$ & $(8.18,9.53)$ & $(10.57,17.98)$ & 1.144 & 0.034 \\
\hline 10 & $(18.28,13.50)$ & $(14.47,6.54)$ & $(6.03,6.33)$ & $(4.25,9.31)$ & $(10.27,15.64)$ & 1.087 & 0.023 \\
\hline
\end{tabular}

TABLE 9: Analysis of validation results for the proposed model.

\begin{tabular}{lc}
$\overline{\overline{F\left(X_{i}^{*}\right)}}$ & 1.153 \\
$\left|\overline{F\left(X_{i}^{*}\right)}-E F\left(X_{i}^{*}\right)\right|$ & 0.043 \\
$\frac{\left|E F\left(X_{i}^{*}\right)-\overline{F\left(X_{i}^{*}\right)}\right|}{E F\left(X_{i}^{*}\right)}$ & $3.87 \%$ \\
\hline
\end{tabular}

its allocated area. Table 7 presents the randomly selected areas for validation of the proposed model. In each of the scenarios, the actual value of the objective function is computed since the customer location is a point. The maximum capacity percentage of the two new facilities is set as 0.425 and 0.58 , and the optimum expected value of the objective function for the five selected areas is equal to 1.11. The validation results of the proposed model are tabulated in Table 8 . The actual value of the objective function for each scenario is listed in column 7; column 8 shows the error of our model, which is the difference between the actual value in column 7 and the expected value of the objective function for the five selected regions.

The final analysis of the model validation is reported in Table 9. The first row is related to the average of the objective function values for the ten scenarios which are extracted from column 7 of Table 8 . The second row indicates the average error between the expected objective function value of the proposed model, and the obtained average amount in the first row. The average error percentage of the model is presented in the last row. As can be seen in Table 9, the average error percentage is equal to $3.87 \%$ which shows that the proposed CFLAP model is a good estimator of the actual objective function value and its solution is near to optimal. Hence, the proposed stochastic model is valid.

In order to highlight the advantages of the proposed model as compared to the most relevant existing literature, a comparison has been made with the model developed by Canbolat and Wesolowsky [47]. The results are summarized in Table 10.
As shown, the work of Canbolat and Wesolowsky [47] was dedicated to a single facility location problem where deterministic demand coordinates were utilized. In addition, their model was tested using a numerical example. In contrast, this study has developed a stochastic model that considers probabilistic demand locations for a multifacility locationallocation problem. The applicability of the proposed model has been evaluated using an actual case study, thus justifying its usefulness in the real-world context.

\section{Conclusions}

In this study, we have considered the planar center locationallocation problem for locating some emergency facilities in the presence of a fixed line barrier in a region. Although some studies have been done on facility location problems with barriers, little attention has been devoted to the probabilistic formulation of these problems where demand coordinates are distributed according to a bivariate probability distribution. In this paper, we have proposed a MINLP model for CFLAP by considering probabilistic demand regions and a fixed line barrier simultaneously, thus making the model more realistic. This study aims at finding the optimum locations of two relief rooms and their corresponding allocation which leads to minimizing the maximum expected weighted distance from the relief rooms to all the demand regions. A real-world case study has been carried out in order to show the effectiveness of the proposed model and the problem has been solved by the GAMS 22.1 software in order to find the optimum solution.

As an approximation, uniform distributions have been used to represent demand region locations. Thus, one possible area for future research is to formulate the problem with the exact probability distributions based on goodness-of-fit tests. In addition, considering the minimax regret objective function for hazardous location problems is another possible extension that can be studied. 
TABLE 10: Comparison results.

\begin{tabular}{ll}
\hline Canbolat and Wesolowsky [47] & This study \\
\hline Deterministic coordinates of demand locations & Stochastic coordinates of demand locations \\
Single facility location & Multifacility location \\
Numerical example & Real-world application \\
\hline
\end{tabular}

\section{Conflict of Interests}

The authors declare that there is no conflict of interests regarding the publication of this paper.

\section{References}

[1] H. W. Hamacher and S. Nickel, "Restricted planar location problems and applications," Naval Research Logistics, vol. 42, no. 6, pp. 967-992, 1995.

[2] K. Klamroth, "Planar weber location problems with line barriers," Optimization, vol. 49, no. 5-6, pp. 517-527, 2001.

[3] A. Krishna, G. S. Dangayach, and R. Jain, "Service recovery: literature review and research issues," Journal of Service Science Research, vol. 3, no. 1, pp. 71-121, 2011.

[4] J. J. Sylvester, "A question in the geometry of situation," Quarterly Journal of Pure and Applied Mathematics, vol. 1, no. 1, pp. 79-80, 1857.

[5] J. Elzinga and D. W. Hearn, "Geometrical solutions for some minimax location problems," Transportation Science, vol. 6, no. 4, pp. 379-394, 1972.

[6] R. L. Francis, L. F. McGinnis Jr., and J. A. White, Facility Layout and Location: An Analytical Approach, Prentice-Hall, Englewood Cliffs, NJ, USA, 2nd edition, 1992.

[7] A. Mehrez, Z. Sinuany-Stern, T. Arad-Geva, and S. Binyamin, "On the implementation of quantitative facility location models: the case of a hospital in a rural region," Journal of the Operational Research Society, vol. 47, no. 5, pp. 612-625, 1996.

[8] M. S. Daskin, S. M. Hesse, and C. S. Revelle, " $\alpha$-Reliable pminimax regret: a new model for strategic facility location modeling," Location Science, vol. 5, no. 4, pp. 227-246, 1997.

[9] F. Hurtado, V. Sacristán, and G. Toussaint, "Some constrained minimax and maximin location problems," Studies in Locational Analysis, no. 15, pp. 17-35, 2000.

[10] N. Altay and W. G. Green III, "OR/MS research in disaster operations management," European Journal of Operational Research, vol. 175, no. 1, pp. 475-493, 2006.

[11] N. C. Simpson and P. G. Hancock, "Fifty years of operational research and emergency response," Journal of the Operational Research Society, vol. 60, no. 1, pp. S126-S139, 2009.

[12] S. Ichoua, "Relief distribution networks: design and operations," in Supply Chain Optimization, Design, and Management: Advances and Intelligent Methods, I. Minis, V. Zeimpekis, G. Dounias, and N. Ampazis, Eds., pp. 171-186, IGI Global, Hershey, Pa, USA, 2011.

[13] D. Shishebori and M. S. Jabalameli, "Improving the efficiency of medical services systems: a new integrated mathematical modeling approach," Mathematical Problems in Engineering, vol. 2013, Article ID 649397, 13 pages, 2013.

[14] A. M. Caunhye, X. Nie, and S. Pokharel, "Optimization models in emergency logistics: a literature review," Socio-Economic Planning Sciences, vol. 46, no. 1, pp. 4-13, 2012.
[15] G. Galindo and R. Batta, "Review of recent developments in OR/MS research in disaster operations management," European Journal of Operational Research, vol. 230, no. 2, pp. 201-211, 2013.

[16] H. D. Sherali, T. B. Carter, and A. G. Hobeika, "A locationallocation model and algorithm for evacuation planning under hurricane/flood conditions," Transportation Research Part B, vol. 25, no. 6, pp. 439-452, 1991.

[17] M.-S. Chang, Y.-L. Tseng, and J.-W. Chen, "A scenario planning approach for the flood emergency logistics preparation problem under uncertainty," Transportation Research Part E, vol. 43, no. 6, pp. 737-754, 2007.

[18] J.-B. Sheu, "An emergency logistics distribution approach for quick response to urgent relief demand in disasters," Transportation Research Part E, vol. 43, no. 6, pp. 687-709, 2007.

[19] H. O. Mete and Z. B. Zabinsky, "Stochastic optimization of medical supply location and distribution in disaster management," International Journal of Production Economics, vol. 126, no. 1, pp. 76-84, 2010.

[20] W. Jiang, L. Deng, L. Chen, J. Wu, and J. Li, "Risk assessment and validation of flood disaster based on fuzzy mathematics," Progress in Natural Science, vol. 19, no. 10, pp. 1419-1425, 2009.

[21] C. G. Rawls and M. A. Turnquist, "Pre-positioning of emergency supplies for disaster response," Transportation Research Part B, vol. 44, no. 4, pp. 521-534, 2010.

[22] P. Yi, S. K. George, J. A. Paul, and L. Lin, "Hospital capacity planning for disaster emergency management," Socio-Economic Planning Sciences, vol. 44, no. 3, pp. 151-160, 2010.

[23] R. Halper and S. Raghavan, "The mobile facility routing problem," Transportation Science, vol. 45, no. 3, pp. 413-434, 2011.

[24] L. Wu, Q. Tan, and Y. Zhang, "Delivery time reliability model of logistic network," Mathematical Problems in Engineering, vol. 2013, Article ID 879472, 5 pages, 2013.

[25] J. Xu, J. Gang, and X. Lei, "Hazmats transportation network design model with emergency response under complex fuzzy environment," Mathematical Problems in Engineering, vol. 2013, Article ID 517372, 22 pages, 2013.

[26] S. Duran, M. A. Gutierrez, and P. Keskinocak, "Pre-positioning of emergency items for CARE international," Interfaces, vol. 41, no. 3, pp. 223-237, 2011.

[27] W. Klibi, S. Ichoua, and A. Martel, "Prepositioning emergency supplies to support disaster relief: a stochastic programming approach," Centre interuniversitaire de recherche sur les réseaux d'entreprise, la logistique et le transport (CIRRELT), Canada, 2013.

[28] L. Cooper, “A random locational equilibrium problem," Journal of Regional Sciences, vol. 14, no. 1, pp. 47-54, 1974.

[29] I. N. Katz and L. Cooper, "An always-convergent numerical scheme for a random locational equilibrium problem," SIAM Journal of Numerical Analysis, vol. 11, no. 4, pp. 683-692, 1974.

[30] G. O. Wesolowsky, "The weber problem with rectangular distances and randomly distributed destinations," Journal of Regional Sciences, vol. 17, no. 1, pp. 53-60, 1977. 
[31] R. Carbone and A. Mehrez, "The single facility minimax distance problem under stochastic location of demand," Management Science, vol. 26, no. 1, pp. 113-115, 1980.

[32] O. Berman, J. Wang, Z. Drezner, and G. O. Wesolowsky, "A probabilistic minimax location problem on the plane," Annals of Operations Research, vol. 122, no. 1-4, pp. 59-70, 2003.

[33] I. Averbakh and S. Bereg, "Facility location problems with uncertainty on the plane," Discrete Optimization, vol. 2, no. 1, pp. 3-34, 2005.

[34] A. Foul, "A 1-center problem on the plane with uniformly distributed demand points," Operations Research Letters, vol. 34, no. 3, pp. 264-268, 2006.

[35] B. Pelegrín, J. Fernández, and B. Tóth, “The 1-center problem in the plane with independent random weights," Computers \& Operations Research, vol. 35, no. 3, pp. 737-749, 2008.

[36] E. Durmaz, N. Aras, and İ. K. Altınel, "Discrete approximation heuristics for the capacitated continuous location-allocation problem with probabilistic customer locations," Computers \& Operations Research, vol. 36, no. 7, pp. 2139-2148, 2009.

[37] M. S. Canbolat and M. Von Massow, "Locating emergency facilities with random demand for risk minimization," Expert Systems with Applications, vol. 38, no. 8, pp. 10099-10106, 2011.

[38] S. A. Hosseinijou and M. Bashiri, "Stochastic models for transfer point location problem," International Journal of Advanced Manufacturing Technology, vol. 58, no. 1-4, pp. 211-225, 2012.

[39] I. N. Katz and L. Cooper, "Facility location in the presence of forbidden regions, I: formulation and the case of Euclidean distance with one forbidden circle," European Journal of Operational Research, vol. 6, no. 2, pp. 166-173, 1981.

[40] K. Klamroth and M. M. Wiecek, "A bi-objective median location problem with a line barrier," Operations Research, vol. 50, no. 4 , pp. 670-679, 2002.

[41] Y. P. Aneja and M. Parlar, "Algorithms for weber facility location in the presence of forbidden regions and/or barriers to travel," Location Science, vol. 3, no. 2, pp. 134-134, 1995.

[42] S. E. Butt and T. M. Cavalier, "An efficient algorithm for facility location in the presence of forbidden regions," European Journal of Operational Research, vol. 90, no. 1, pp. 56-70, 1996.

[43] R. G. McGarvey and T. M. Cavalier, "A global optimal approach to facility location in the presence of forbidden regions," Computers and Industrial Engineering, vol. 45, no. 1, pp. 1-15, 2003.

[44] K. Klamroth, "Algebraic properties of location problems with one circular barrier," European Journal of Operational Research, vol. 154, no. 1, pp. 20-35, 2004.

[45] M. Bischoff and K. Klamroth, "An efficient solution method for weber problems with barriers based on genetic algorithms," European Journal of Operational Research, vol. 177, no. 1, pp. 2241, 2007.

[46] M. Bischoff, T. Fleischmann, and K. Klamroth, "The multifacility location-allocation problem with polyhedral barriers," Computers \& Operations Research, vol. 36, no. 5, pp. 1376-1392, 2009.

[47] M. S. Canbolat and G. O. Wesolowsky, "A planar single facility location and border crossing problem," Computers \& Operations Research, vol. 39, no. 12, pp. 3156-3165, 2012.

[48] S. Huang, R. Batta, K. Klamroth, and R. Nagi, "The Kconnection location problem in a plane," Annals of Operations Research, vol. 136, no. 1, pp. 193-209, 2005.

[49] P. Nandikonda, R. Batta, and R. Nagi, "Locating a 1-center on a Manhattan plane with "arbitrarily" shaped barriers," Annals of Operations Research, vol. 123, no. 1-4, pp. 157-172, 2003.
[50] P. M. Dearing, K. Klamroth, and R. Segars Jr., "Planar location problems with block distance and barriers," Annals of Operations Research, vol. 136, no. 1, pp. 117-143, 2005.

[51] A. Sarkar, R. Batta, and R. Nagi, "Placing a finite size facility with a center objective on a rectangular plane with barriers," European Journal of Operational Research, vol. 179, no. 3, pp. 1160-1176, 2007.

[52] L. Friebß, K. Klamroth, and M. Sprau, "A wavefront approach to center location problems with barriers," Annals of Operations Research, vol. 136, no. 1, pp. 35-48, 2005.

[53] M. S. Canbolat and G. O. Wesolowsky, "The rectilinear distance weber problem in the presence of a probabilistic line barrier," European Journal of Operational Research, vol. 202, no. 1, pp. 114-121, 2010.

[54] M. S. Canbolat and G. O. Wesolowsky, "On the use of the Varignon frame for single facility weber problems in the presence of convex barriers," European Journal of Operational Research, vol. 217, no. 2, pp. 241-247, 2012.

[55] A. R. Gharagozlou, A. R. A. Ardalan, and M. H. Diarjan, "Environmental planning for disaster management by using GIS, (a case study about flood in Mazandaran)," The International Archives of the Photogrammetry, Remote Sensing and Spatial Information Sciences, vol. 37, B8, pp. 323-326, 2008.

[56] N. Sahinidis and M. Tawarmalani, "Baron," in GAMS-The Solver Manuals, pp. 19-32, GAMS Development Corporation, Washington, DC, USA, 2008. 


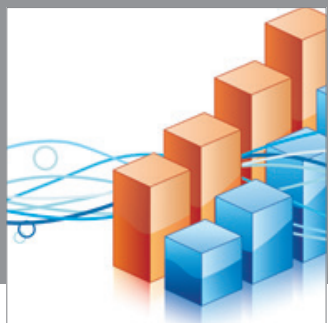

Advances in

Operations Research

mansans

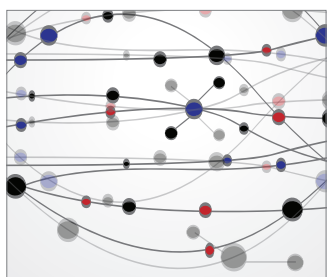

The Scientific World Journal
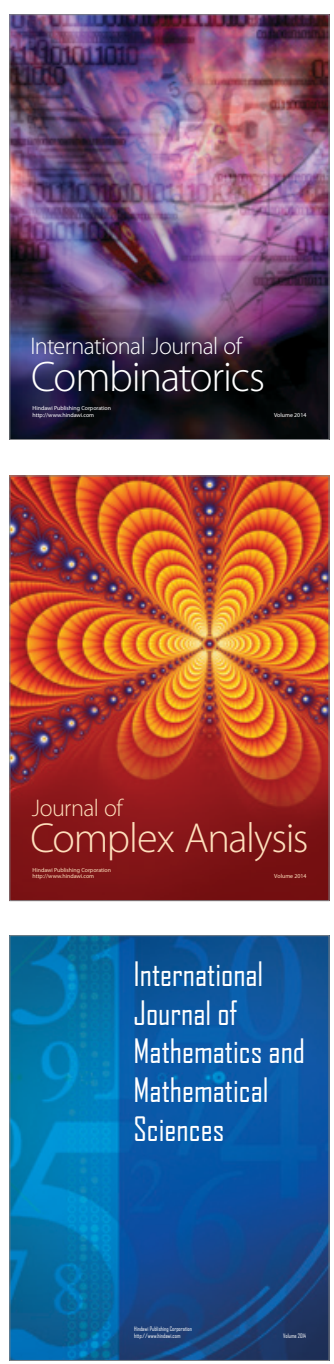
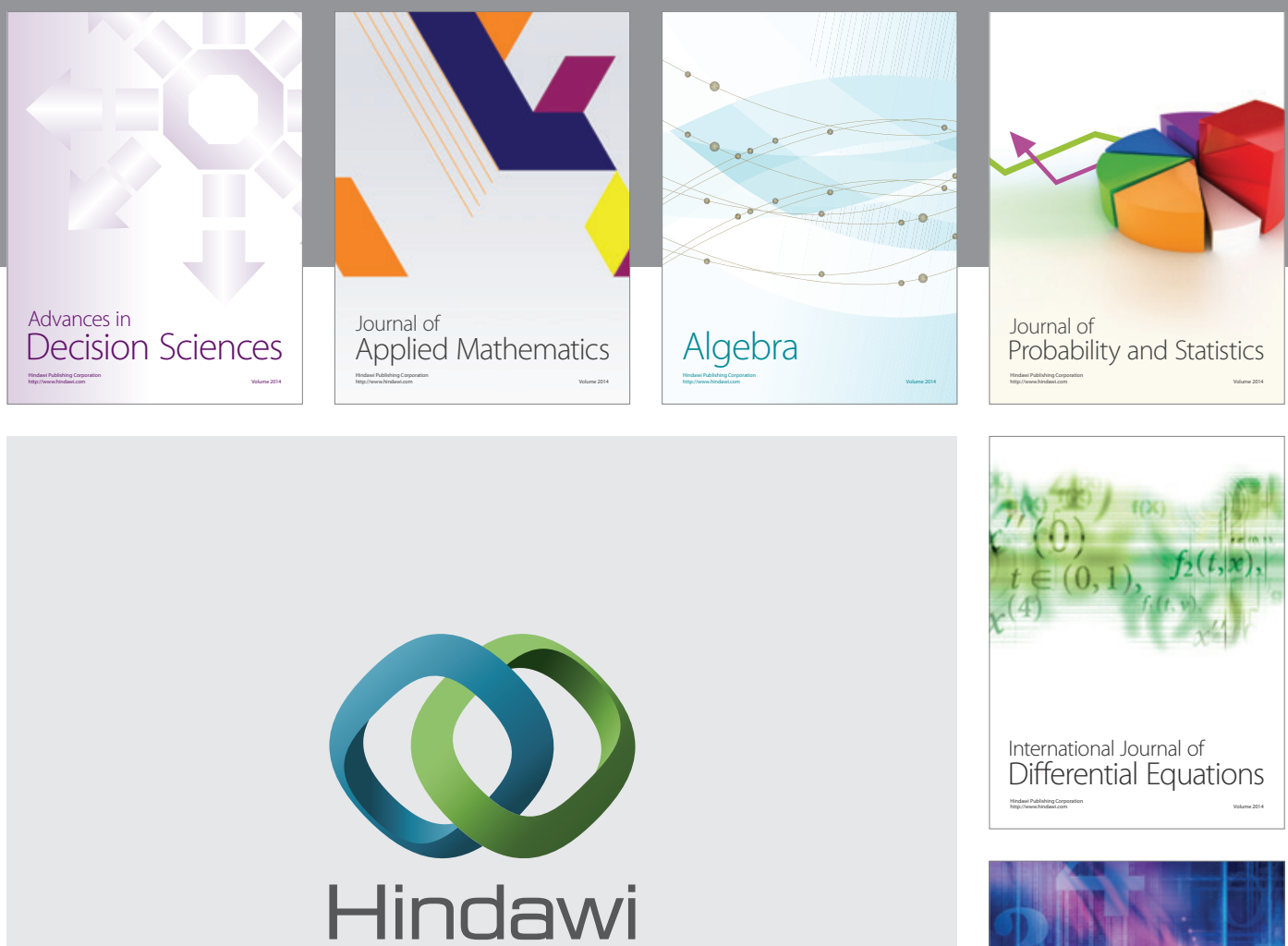

Submit your manuscripts at http://www.hindawi.com
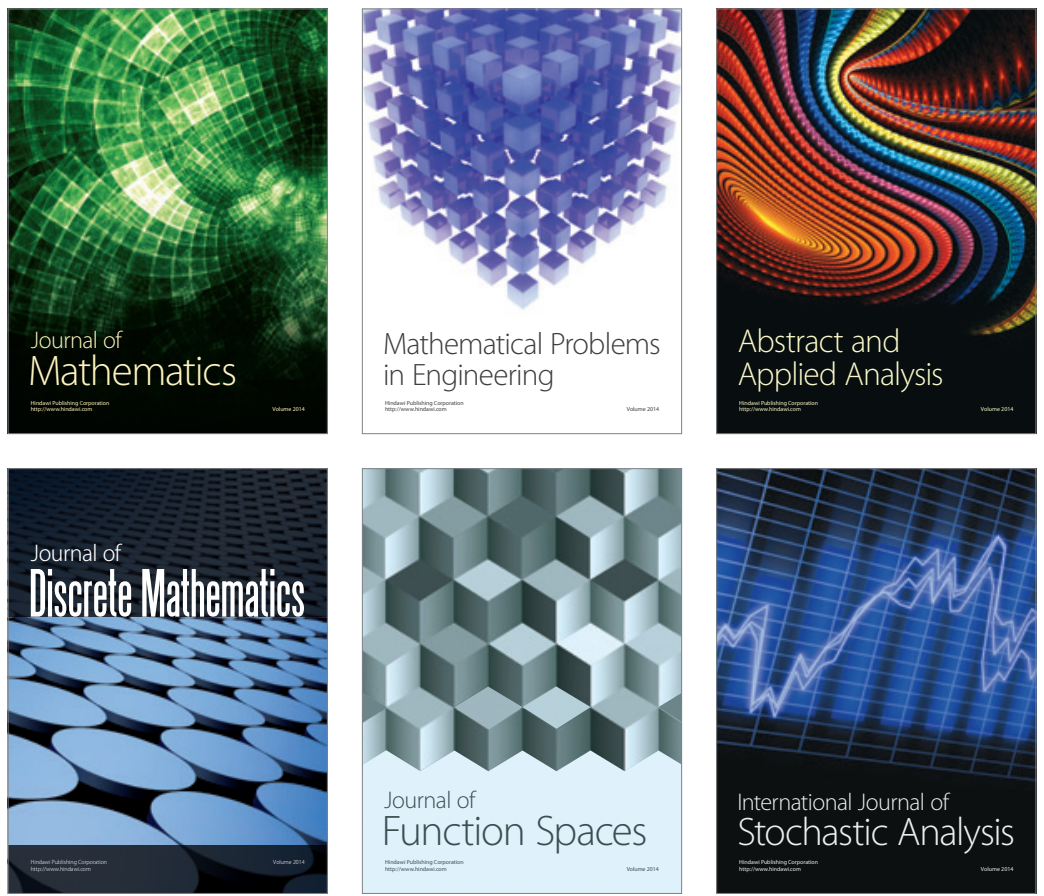

Journal of

Function Spaces

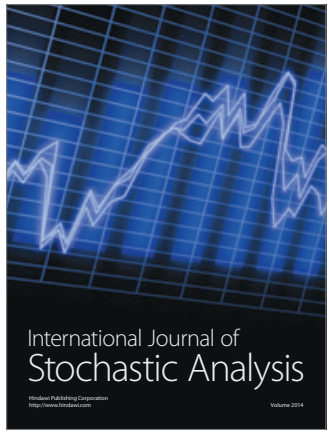

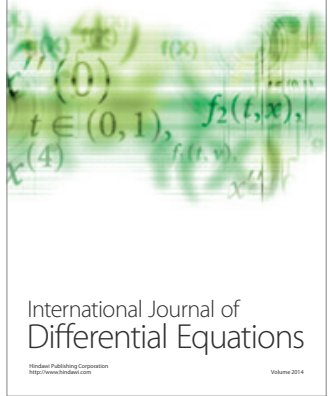
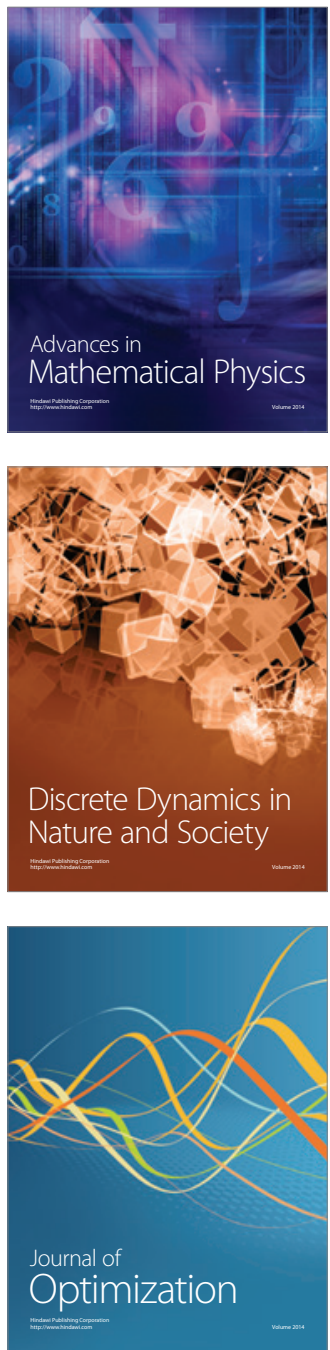